\title{
NEW HOM COUPLER DESIGN FOR 3.9 GHZ SUPERCONDUCTING CAVITIES AT FNAL
}

\author{
Timergali Khabiboulline, Ivan Gonin, Nikolay Solyak. FNAL, Batavia, IL 60510, U.S.A
}

\section{Abstract}

Over the last few years Fermilab has developed the superconducting third harmonic section for $\mathrm{t}$ he FLASH (TTF/DESY) upgrade. Initial v ertical tests of 9 -cell $\mathrm{Nb}$ cavities didn't reach the designed accelerating gradient. The main factor of gradient limitation was multipacting in the HOM coupler. In this paper we present the results of vertical tests ac companied with 3D analysis simulations of multipacting. Also we discuss the RF design of the new HOM couplers. The goal of the new design is to eliminate multipacting and to increase the second resonance frequency of the HOM coupler. Increasing this frequency reduces electric and magnetic fields , resulting in decreased thermal load on the antenna. Two cavities with modified HOM couplers have achieved operating gradients of $23 \mathrm{MV} / \mathrm{m}$.

\section{INTRODUCTION}

Large beam current operation of $\mathrm{t}$ he $3{ }^{\text {rd }}$ harmonic 3.9 $\mathrm{GHz}$ cavity imposes limits on beam excitation of the high order modes (HOM), Fig.1. Two HOM couplers located in beam pipes on each end of the cavity should help to extract HOM power from the cavity and reduce quality factor $\mathrm{Q}$ of the cavity for these modes. On the other hand these HOM couplers should not extract too much power from the cavity at operating mode.

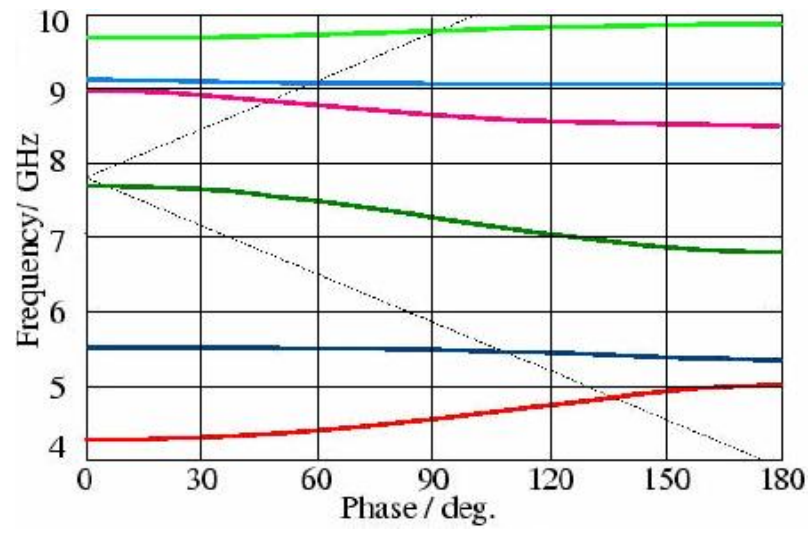

Figure 1: $1^{\text {st }}$ to $6^{\text {th }}$ dipole pass bands of the $3^{\text {rd }}$ harmonic Cavity.

External Q of the power coupler is about Qext $=10^{6}$ and the power ex cited by the beam is about $50 \mathrm{~kW}$. Power leakage through each HOM coupler should be less than 5 $\mathrm{W}$, so external quality factor of each HOM coupler for these modes should be more than Qext $>10^{10}$. The first dipole HOM pass band (frequencies 4.2 $-5.0 \mathrm{GHz}$ ) with very high shunt impedance (see Figure 2 ) is close to the operating mode frequency $3.9 \mathrm{GH}$ z. External Q of the HOMs at 4.7 GHz should be less than $10 \quad 4$. Cut-off frequency of the beam pipe is $4.4 \mathrm{GHz}$ and some modes can be trapped in one cavity. The HOM coupler should fit all these requirements.

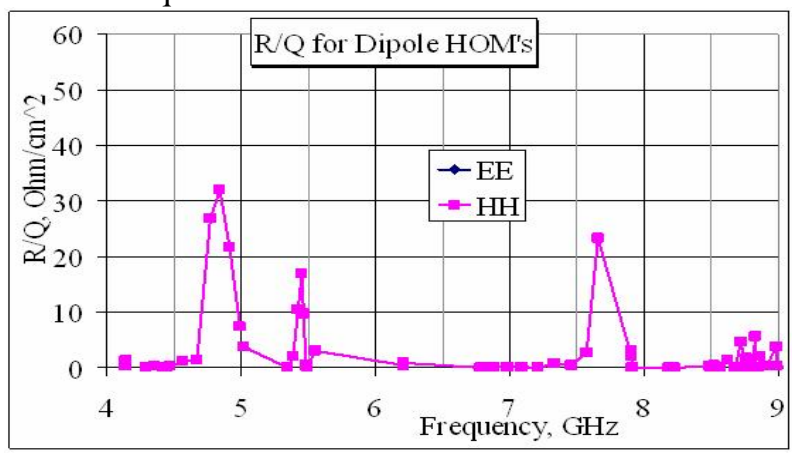

Figure 2: Calculated R/Q of the 9 cell $\quad 3^{\text {rd }}$ harmonic Cavity.

\section{PROBLEMS WITH HOMS}

\section{Test of the 9-cell cavity \#2.}

During the high power tests of cavity \#2 thermal breakdown in the HOM coupler with relatively low field in the cavity was found, corresponding to Eacc=1 -2 $\mathrm{MV} / \mathrm{m}$ accelerating field. Multipacting analysis of the HOM coupler predicts two MP zones. The first one is located in the tuning gap at Eacc $=1.5 \mathrm{MV} / \mathrm{m}, \quad$ and the second one is wider and stronger at Eacc $=12-19 \mathrm{MV} / \mathrm{m}$ [1-3].

After multiple tests with $\sim 150 \mathrm{~W}$ power $\mathrm{CW}$ the Fprobes of both HOMs were fractured, as shown in Figure 3. Thermal and stress analyses sh ow stresses near the yield point. Multiple heating cycles caused by RF power loss and cooling between pulse s can result in mechanical fracture [1].

The first buffered chemical polish ( BCP) o n cavity \#2 was not properly ex ecuted. Because this could increa se the secondary electron emission coefficient and contribute to multipacting, it was decided to proceed with production and test of cavity \#3.

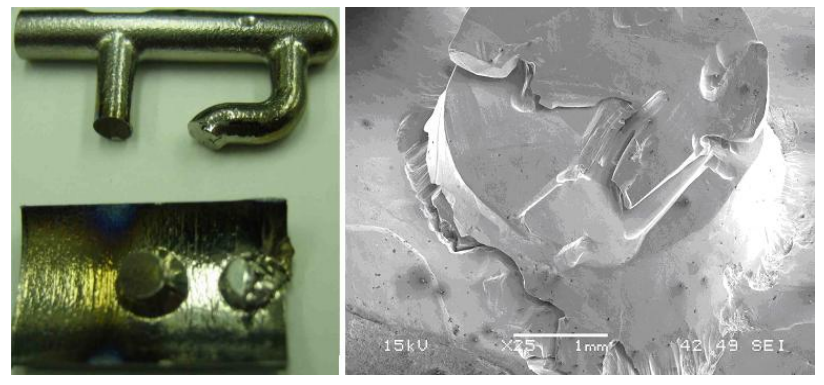

Figure 3: Curved leg of the HOM F-probe fractured from HOM can. 


\section{Test of the 9-cell cavity \#3.}

We did not s ee multipacting in low field regimes as we did in cavity \#1 but $\mathrm{s}$ ignificant multipac ting activity appears with cavity gradient $\mathrm{s}$ above $11 \mathrm{MV} / \mathrm{m} . \mathrm{T}$ he highest accelerating field level achieved in cavity \#3 is 12 $\mathrm{MV} / \mathrm{m}$, limited by quench in the HOM couplers.

\section{NEW HOM COUPLER RF DESIGN}

Analysis of the thermal breakdown problem in cavity \#2 and cavity \#3 shows a multipacting problem inside the HOM coupler. Multipacting conditions exist only in some levels of the surface fields and reducing surface field should help damp multipacting. The f ield level in the original HOM design is fairly high since the coupler's resonance is close to the cavity's operating frequency. Our strategy of re -designing the HOM coupler was to reduce fields in the coupler at $3.9 \mathrm{GHz}$ without degradation of damping performance for all high modes. This can be done by shifting coupler resonance frequency out of operating frequency.

The new HOM design should eliminate sever al identified problems such as, multipacting near the welding joints and tuning gap, and reduce fields in the $\mathrm{HOM}$ at $3.9 \mathrm{GHz}$. Also, i ncreasing the leg and probe diameters improves thermal conductance, reducing operating temperature at the probe tip. Some RF models of several possible versions are shown in Fig. 4. Version1 is the original design included in the first three cavities.

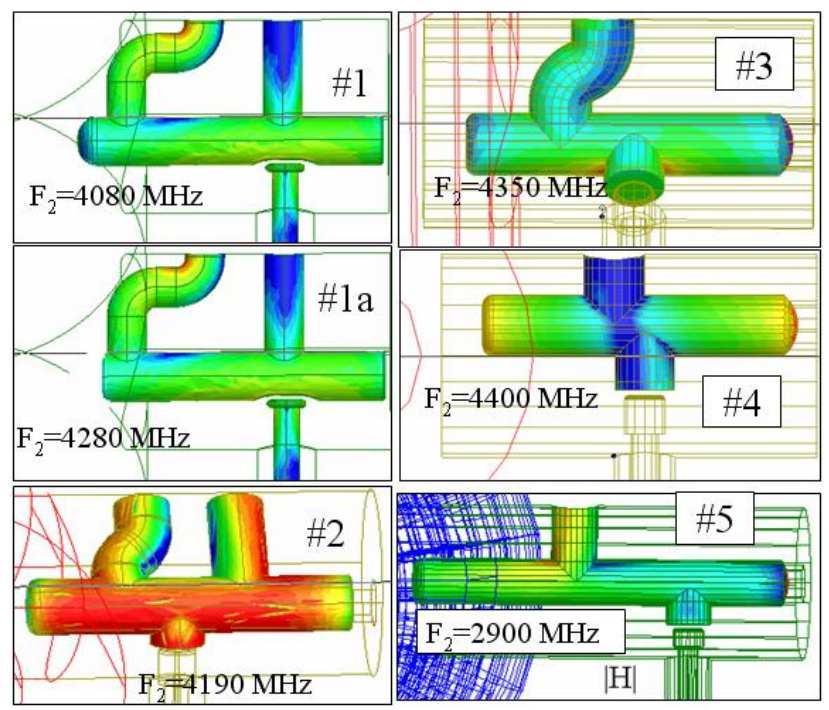

Figure 4: Different HOM coupler designs. Calculated fields on the F-probe surface are shown.

Version-2 has a $6 \mathrm{~mm}$ F-probe diameter (increased from $4 \mathrm{~mm}$ ) to improve cooling, a "straightened" 1 st p ost, and the tuning gap increased from $0.5 \mathrm{~mm}$ to $2 \mathrm{~mm}$ Additionally, a boss was added to the probe adjacent to the antenna to reduce heating ca used by RF power losses because there are high magnetic fields in the antenna location. The HOM coupler antenna is located in the minimum of the electric field to reduce coupling in notch frequency $3.9 \mathrm{GHz}$. The tuning gap was increased to reduce multipacting. To keep notch frequency at $3.9 \mathrm{GHz}$, the probe length between the leg and tuning gap was also increased.

Version-3 has one curved post similar to that in version- 2 but the second post is eliminated to try to reduce therma 1 stresses. Version- 4 has a single straight post. These two single-post versions have very small magnetic coupling and mostly electric coupling $\mathrm{w}$ ith the cavity HOM modes . HOM version-4 has lower surface fields and therefore is more preferable. See Table 1.

Table 1: Surface fields compared with the initial design.

\begin{tabular}{|c|c|c|c|}
\hline Version & $\mathrm{Hp} / \mathrm{Hp} \# 1$ & $\mathrm{Ep} / \mathrm{Ep} \# 1$ & $\mathrm{~F} 2-\mathrm{F} 0, \mathrm{MHz}$ \\
\hline HOM-1 & 1 & 1 & 180 \\
\hline HOM-1a & $\mathbf{0 . 4}$ & $\mathbf{0 . 4}$ & $\mathbf{3 8 0}$ \\
\hline HOM-2 & $\mathbf{0 . 7 6}$ & $\mathbf{0 . 4 5}$ & $\mathbf{2 9 0}$ \\
\hline HOM-3 & 0.77 & .48 & 450 \\
\hline HOM-4 & $\mathbf{0 . 6 7}$ & $\mathbf{0 . 3 1}$ & $\mathbf{5 0 0}$ \\
\hline HOM-5 & 0.57 & 0.098 & -1000 \\
\hline Cavity & $\mathbf{7 . 4}$ & $\mathbf{3 . 5}$ & $\mathbf{0}$ \\
\hline
\end{tabular}

Version-5 is also a single post design . The $m$ ain difference from the other designs is that the $2^{\text {nd }}$ resonance frequency $\mathrm{F}_{2}=2.9 \mathrm{GHz}$ of the HOM coupler moved to a position lower than the operating frequency of the cavity $\mathrm{F}_{0}=3.9 \mathrm{GHz}$. (Figure 5)

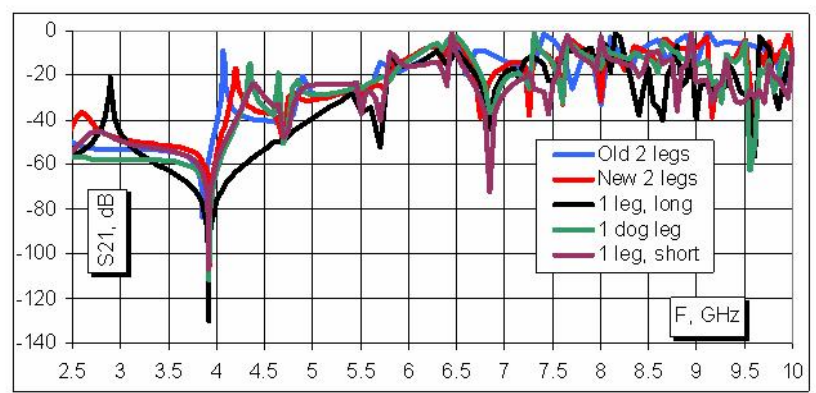

Figure 5: S21 from beam pipe to HOM.

HOM version-1a is a modification of the initial design with a trimmed F-probe tip. Trimming reduces surface fields by $\sim 2.5$ times c ompared with the initial design. All presented designs show similar damping performances at high frequencies $>5 \mathrm{GHz}$ as shown on transition plot in Figure 5. HFSS calculations do not show degradation of coupling for the $1^{\text {st }}$ and $2^{\text {nd }}$ dipole bands (Figure 6).

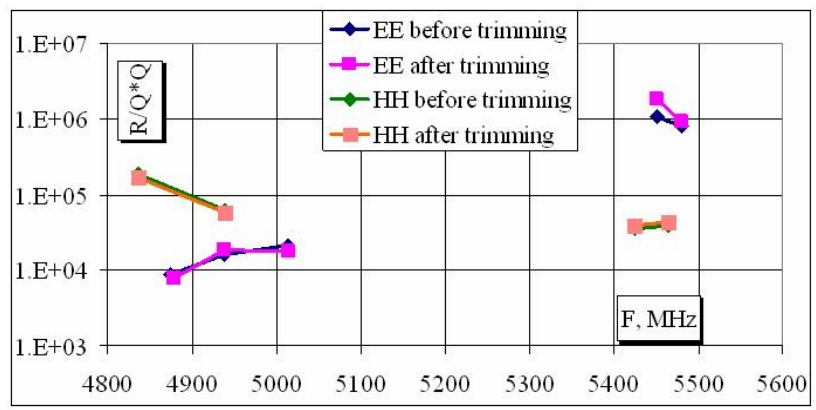

Figure 6: Shunt Impedance calculations for version-1 and 1a. 
Based on simulated performance we built and tested copper prototypes of version -2 and 4 . At the time we already had finished three pairs of cavity end assemblies with HOM version-1 welded in. It was dec ided that the fastest way to have good cavity to test was to trim off the tips of one pair , resulting in HOM version -1a and finish cavity \#4 with them.

\section{Copper mockups of HOM version-2 and 4.}

Two copper mockups of each type of the HOM coupler version-2 and 4 were machined and brazed (Figure 7). We already had two full -size copper 9 -cell cavities for RF measurements. Mockup HOM couplers were attached to the copper cavity with rotat able flanges. Notch frequency of the HOM coupler depends on angular position o $\mathrm{f}$ the HOM coupler and RF field pattern in the beam pipe. Field patterns in the beam pipe depend on power excitation from the power coupler and cavity beam loading.

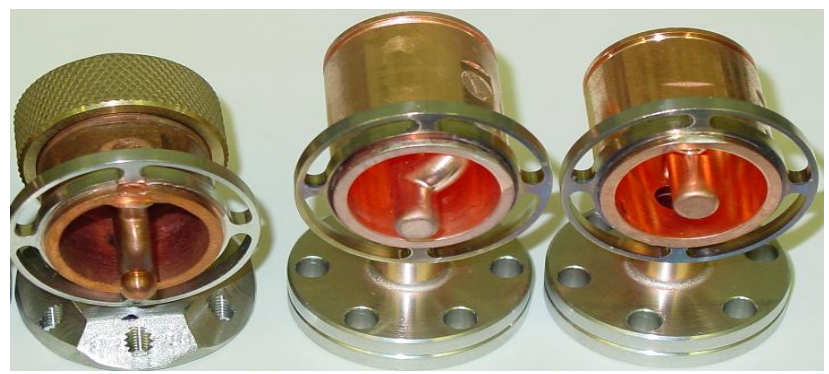

Figure 7: Copper mockups of the HOM couplers. Left is old design, in the middle version -2 , right version -4 .

A series of calculations and measurements were done to find the optimum angular position of the HOM coupler. The o ptimum position is found when coupling of the HOM coupler with the cavity and main coupler with the HOM coupler are both minimum. Calculations and measurements are in a good agreement . HOM version-4 shows two optimum position $\mathrm{s}$ at 130 degree $\mathrm{s}$ and -50 degrees (Figure 8). Because of lower notch frequ ency and preferable mechanical design , -50 degree position was chosen for cavity production.

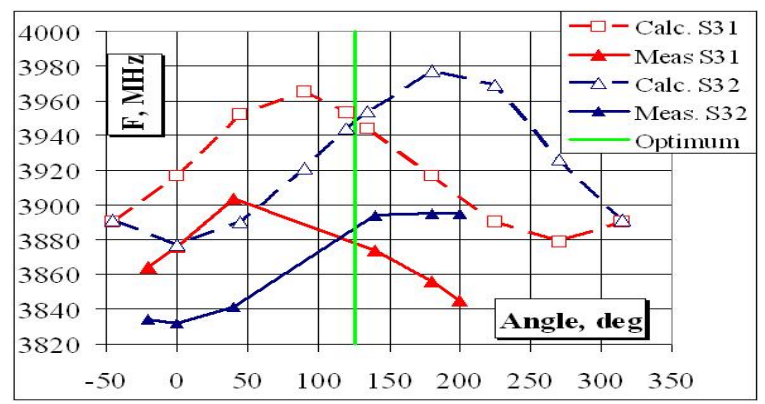

Figure 8: Notch frequency vs. position of the HOM coupler version-4.

\section{Test of the 9-cell cavities \#4 and modified \#3.}

Cavity \#4 was manufactured with HOM version -1a, trimmed HOM F-probe tips. Several cold tests were done and $23 \mathrm{MV} / \mathrm{m}$ accel erating field gradients were reached without thermal breakdown in the HOMs. One test was done with the HOM antennas installed and $22 \mathrm{MV} / \mathrm{m}$ gradient reached for a pulsed regime and $12 \mathrm{MV} / \mathrm{m}$ in $\mathrm{CW}$ regime.

Cavity \#3 with original design HOM version-1 showed MP problems in vertical tests, Eacc was limited $\sim 12$ $\mathrm{MV} / \mathrm{m}$. The cavity was then modified by trimming the tips (HOM version-1a) and retested again. In two consecutive tests Eacc $=23 \mathrm{MV} / \mathrm{m}$ was reached.

\section{SIMULATIONS OF MULTPACTING IN THE HOM COUPLER}

Simulations show multipacting in the tuning $2 \mathrm{~mm}$ gap. This problem was eliminated in new design by rounding the F-probe end near the gap. The results of calculation does not show any problem in the HOM version-2 and 4 in the range of accelerating fields of the cavit $y$ up to 15 $\mathrm{MV} / \mathrm{m}$. Very narrow and low intensity o ne-point MP on the HOM coupler F-probe version-2 at Eacc $~ 7.5 \mathrm{MV} / \mathrm{m}$ (Fig. 9 left) seems acceptable. For HOM version-4 there is no multipacting effect at all (Fig. 9 right).

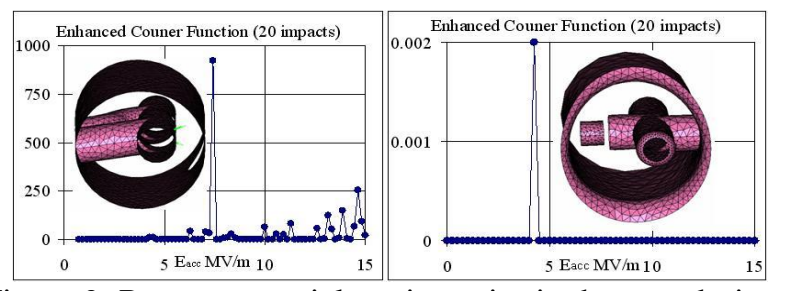

Figure 9: Resonant particle traj ectories in the new design $\mathrm{s}$ version- 2 and version-4 of HOM coupler.

\section{CONCLUSIONS}

F-probe failures in the $3.9 \mathrm{GHz} \mathrm{HOM}$ coupler forced a redesign. A few new designs were proposed. Two cavities with re -designed HOM couplers (trimmed tips) were successfully tested with accelerating field level s reaching $23 \mathrm{MV} / \mathrm{m}$ without any problems . One of them tested in $\mathrm{CW}$ regime up to $12 \mathrm{MV} / \mathrm{m}$. Another two cavities with the same probe design are fabricated and will be tested in 2-3 weeks. Other cavities with HOM coupler version -4 are currently under production.

\section{ACKNOWLEDGEMENTS}

The authors thank Don Mitchell, Mike Foley , Tug Arkan, and Harry Carter for their useful discussions.

\section{REFERENCES}

[1] N.Solyak, et.al, "Production and test results of SC 3.9 $\mathrm{GHz}$ accelerating Cavity at Fermilab", ASC2006.

[2] T Khabiboulline et.al, " "3.9 GHz Superconducting Accelerating 9 -cell cavity vertical test results", this conference, WEPMN111.

[3] I.Gonin, et.al, "Multipactor simulations in superconducting cavities", this conference, WEPMN093. 